arrays by decomposing recrystallized silver phenylacetylide nanowires, as they reported in the September 19 issue of Chemistry of Materials (DOI: 10.1021/ $\mathrm{cm} 071688 \mathrm{i}$ ).

The researchers succeeded in recrystallizing silver phenylacetylide by using the ligand dissociation of $\mathrm{Me}_{3} \mathrm{P}-\mathrm{Ag}-\mathrm{C} \equiv \mathrm{C}-\mathrm{Ph}$. It crystallized as thin wire-shaped crystals because of its highly anisotropic crystalline structure. A fast $\mathrm{PMe}_{3}$ dissociation rate in a high-polarity solvent produced
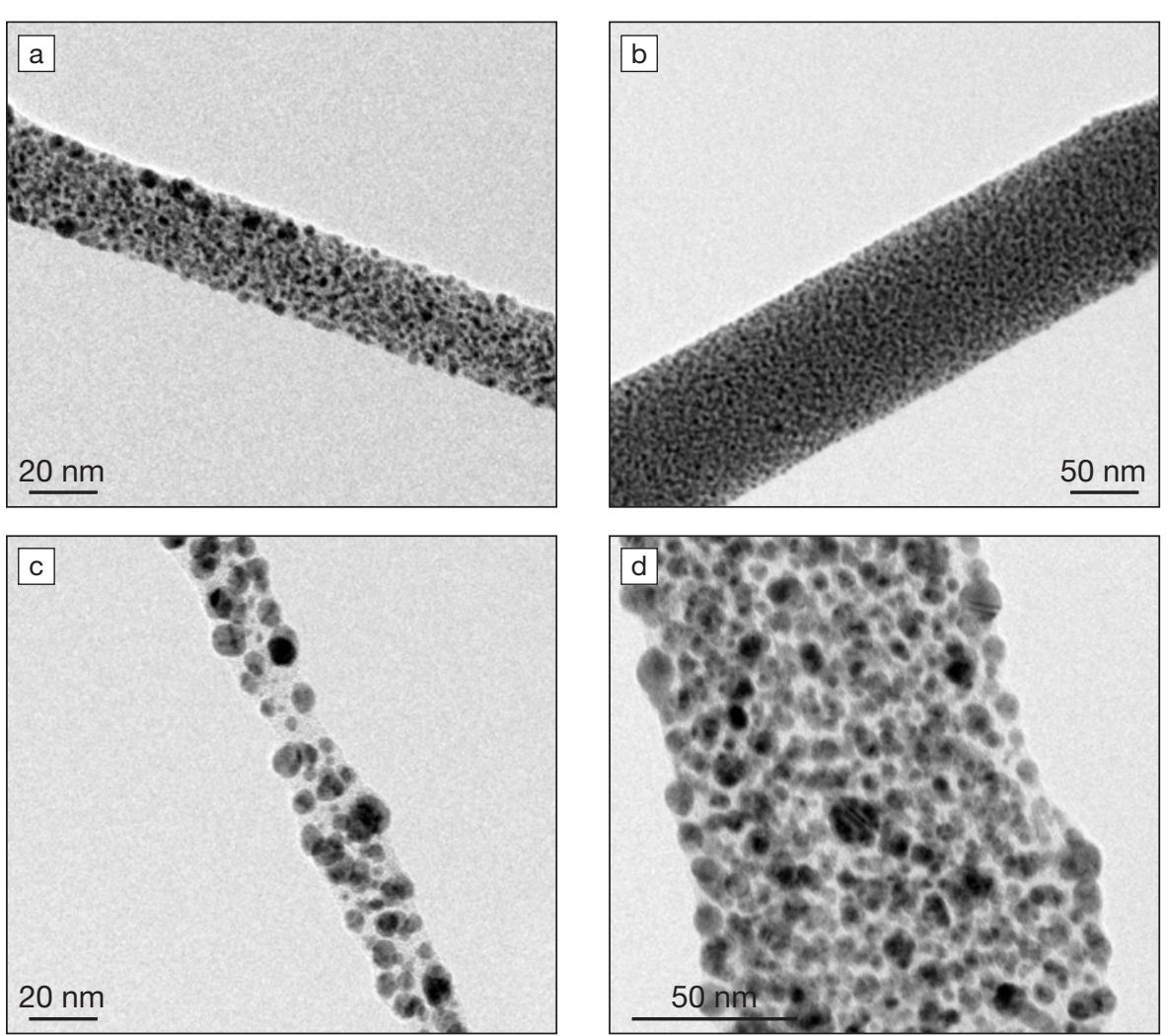

thin silver phenylacetylide nanowires (39 $\pm 11 \mathrm{~nm}$ in $\mathrm{EtOH}$ ), whereas slow crystal growth in a lower-polarity solvent brought thicker nanowires (94 $\pm 12 \mathrm{~nm}$ in $1-\mathrm{BuOH})$. The aspect ratio was $\sim 30-40$, which increased to more than 100 when $\mathrm{CH}_{3} \mathrm{CN}$ was used as a solvent. The strong reducing power of ethynyl anions present in the wires converted the raw material into an assembly of $\mathrm{Ag}$ nanoparticles under mild conditions when they were irradiated with ultraviolet (UV) light,
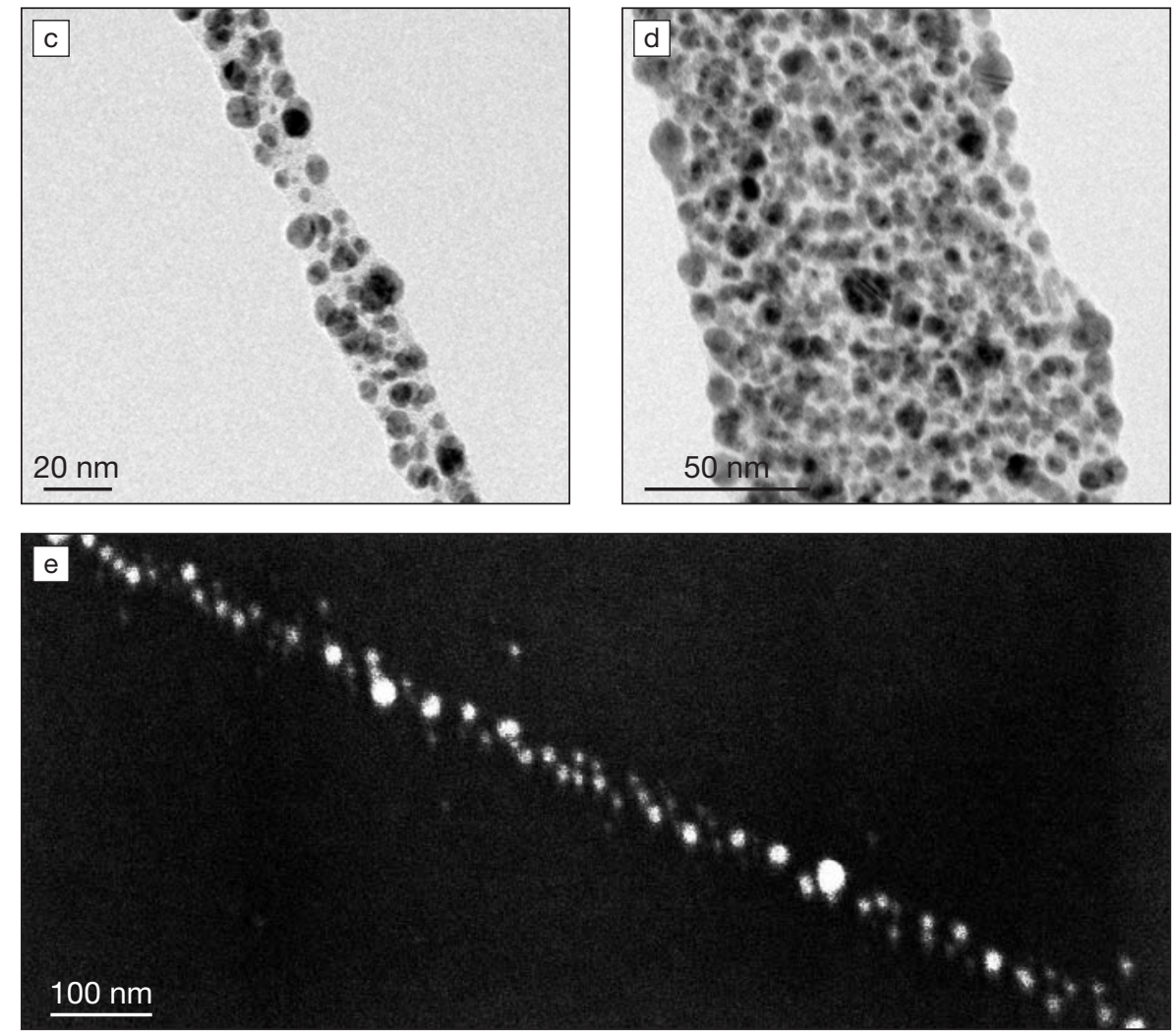

Figure 1. (a-d) Transmission electron microscope images of ultraviolet (UV)-irradiated silver phenylacetylide nanowires: (a) wire/EtOH, 15 min of irradiation; (b) wire/1-BuOH, 15 min of irradiation; (c) wire/EtOH, 3h of irradiation; (d) wire/1-BuOH, 3 h of irradiation. (e) Scanning electron microscope image of decomposed wire/EtOH. Ag nanoparticles are fixed on a Si substrate by heating after UV irradiation. Reprinted with permission from Chemistry of Materials 19(19) (2007) p. 4627. (C2007 American Chemical Society. while the phenylacetylene molecules formed during the decomposition process polymerized. This generated an organic matrix that surrounded the Ag nanoparticles, protecting them from oxidation while keeping the original shape of the wire, without using any template. After 15 minutes of irradiation, the surface plasmon resonance band of $\mathrm{Ag}$ nanoparticles appeared at $485 \mathrm{~nm}$, slightly red-shifted with respect to typical Ag nanoparticles because of the dipole-dipole interaction caused by the short distance between nanoparticles ( $1 \mathrm{~nm}$ for $15 \mathrm{~min}$ of irradiation and $3 \mathrm{~nm}$ for $3 \mathrm{~h}$ of irradiation). Additionally, the high dielectric constant of the matrix can also reduce the plasmon frequency, the researchers said. Particle size was independent of the wire diameter and increased with irradiation time. Transmission electron microscope images recorded by the researchers showed the conversion of a nanowire into a 1D array of homogeneously distributed Ag nanoparticles after irradiation (see Figure 1).

Heating silver phenylacetylide nanowires at $110^{\circ} \mathrm{C}$ for $3 \mathrm{~h}$ was an alternative for obtaining 1D arrays of Ag nanoparticles with a diameter of $2.4 \pm 0.7 \mathrm{~nm}$, similar to that obtained after UV irradiation for $15 \mathrm{~min}$. By using higher temperatures, however, the organic part of the decomposed nanowire melted and evaporated and the Ag nanoparticles spread on the substrate without keeping the 1D array structure. This process was used by the researchers to fix the $1 \mathrm{D} \mathrm{Ag}$ nanoparticle arrays on a substrate after irradiating the original nanowires with UV light.

JOAN J. CARVAJAL

\section{Computational Model Shows Stark Shifts Induce Ultrafast Current in Molecular Wires}

The characterization of molecular wires as essential components of nanoelectronic devices traditionally focuses on electronic transport properties at metal-molecule junctions subject to a bias voltage. Laser radiation with frequency components $\omega$ and $2 \omega$, chosen such that $\omega+2 \omega$ is on or near resonance, are known to induce currents in spatially symmetric systems. In addition to creating laser-induced rectification, the multiphoton absorption transfers electrons into mobile states. At the junctions, however, most molecules exhibit strong vibronic couplings that introduce ultrafast coherence loss and internal relaxation into the electronic dynamics. Recently, however, I. Franco and P. Brumer from the University of Toronto, Canada, and M. Shapiro from Weizmann Institute, Rehovot, Israel, investigated computationally an alternative mechanism that induces 
current in molecular wires with high efficiency, yet is remarkably resistant to the deleterious effects of vibronic coupling.

As reported in the September 21 issue of Physical Review Letters (DOI: 10.1103/ PhysRevLett.99.126802), Franco, Brumer, and Shapiro chose $\omega$ and $2 \omega$ far-off resonance, well below interband transition frequencies, thereby relying on Stark shifts to couple ground and excited electronic states adiabatically. The researchers used a model composed of a trans-polyacetylene (PA) oligomer, the ends of which are connected to macroscopic metallic leads. They employed the so-called tight-binding model (the full Hamiltonian of the system is approximated by the Hamiltonian of an isolated atom centered at each lattice point and the atomic orbitals are assumed to be very small at distances exceeding the lattice constant). The energy of the nuclei and electrons of the oligomer were represented with the well-known Su-SchriefferHeeger (SSH) Hamiltonian, which successfully reproduces the electronic structure and the dynamics of electronic excitations in PA. The electronic structure of the PA chain consists of 50 doubly occupied valence $\pi$ orbitals and 50 empty $\pi^{*}$ states separated by an energy gap of $1.3 \mathrm{eV}$. The leads were treated as rigid, semi-infinite chains. The researchers developed a Hamiltonian for the nanojunctions, treating each as a one-dimensional lattice.

The $\omega+2 \omega$ field was turned on and off smoothly during a 100-fs interval, and the amplitude, $6.1 \times 10^{-3} \mathrm{~V}^{-1}$, was kept constant for $400 \mathrm{fs}$. The photoinduced, electron-vibrational dynamics were followed within a mean-field, mixed quantum-classical approximation. For a relative pulse phase of $\phi_{2 \omega}-2 \phi_{\omega}=0$ and averaging more than 1000 trajectories, the researchers found that the spectrum displayed considerable Stark shifts and narrowing of the energy gap, causing frequent crossings between ground and excited states in individual trajectories. In addition, charge bursts were deposited in the leads when the electron population was transferred from the valence to the conduction band. The researchers observed that a minimum in the energy gap was concomitant with the maximum in the field strength, but the Stark effect was only sufficiently strong to close the energy gap when the field had positive amplitudes. For $\phi_{2 \omega}-2 \phi_{\omega}=\pi / 2$ the researchers found that the Stark shifts were equally strong for positive and negative field amplitudes, resulting in no net current; the direction of the current can therefore be controlled by the relative phase of the laser. Plotting the net rectification as a function of the laser phase, the researchers showed that up to $90 \%$ of the electrons can participate in the net current, the mechanism is resistant to decoherence effects, and the currents observed are phonon-assisted. Franco, Brumer, and Shapiro said that their prediction of ultrafast currents "could lead to the development of molecular switches that operate on a femtosecond timescale."

STEVEN TROHALAKI

\section{Photonic Crystal Enhances Brightness of Quantum Dots}

N. Ganesh, B.T. Cunningham, and colleagues at the University of Illinois, Urbana-Champaign (UIUC) have demonstrated enhanced fluorescence intensity by a factor of up to 108 by placing quantum dots on a photonic crystal. Potential applications include high-brightness light- emitting diodes, optical switches, and personalized, high-sensitivity biosensors.

"We are using photonic crystals in a new way," said Brian Cunningham, a UIUC professor of electrical and computer engineering and corresponding author of an article published in the August 2007 issue of Nature Nanotechnology (p. 515; DOI:10.1038/ nnano.2007.216). "We tune them to the specific wavelength of a laser used to stimulate the quantum dots, which couples the energy more efficiently and increases the brightness."

To enhance the fluorescence of quantum dots, Cunningham and colleagues create plastic sheets of photonic crystal using a
Precision Heating to $1200^{\circ} \mathrm{C}$ in 24 seconds,

\section{for under $\$ 10,000$.}

\section{NOW THAT'S HOT!}

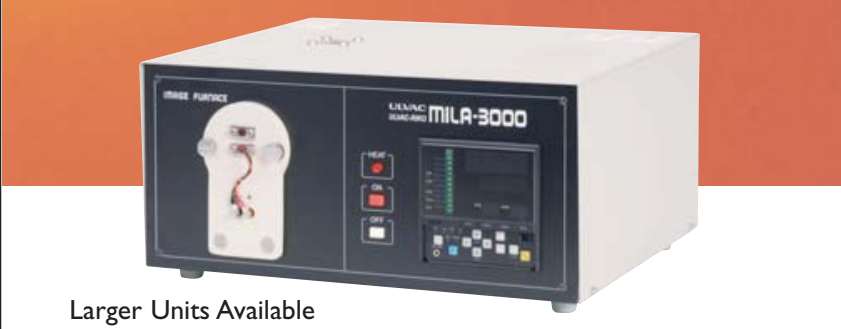

Larger Units Available

Anneal your small samples faster and with better control using the programmable MILA-3000 tabletop furnace. Ulvac's Mini-Lamp Annealing System can rapidly heat and cool samples with its infrared gold reflector furnace, providing precision high temperature control, clean heating and versatile atmosphere selection.

MILA-3000 Features:

- High controlled heating and cooling rates of up to $50^{\circ} \mathrm{C} / \mathrm{s}$

- Sample size $20 \times 20 \times 20 \mathrm{~mm}$

- Air, vacuum and inert/reactive cover gas atmosphere

- Temperature uniformity of $+/-2^{\circ} \mathrm{C}$

- Low power consumption (4 kW)

For all your annealing and thermal processing needs turn up the heat with the MILA-3000!

ULVAC Technologies, Inc Methuen, MA 01844

Phone: 978-686-7550

www.ulvac.com

sales@ulvac.com 